\title{
BMJ Open Effect of high-dose vitamin D3 on 28- day mortality in adult critically ill patients with severe vitamin D deficiency: a study protocol of a multicentre, placebo-controlled double-blind phase III RCT (the VITDALIZE study)
}

\author{
Karin Amrein, ${ }^{1}$ Dhruv Parekh (10 ${ }^{2}$ Sabine Westphal, ${ }^{3}$ Jean-Charles Preiser, ${ }^{4}$ \\ Andrea Berghold, ${ }^{5}$ Regina Riedl, ${ }^{5}$ Philipp Eller (i) , ${ }^{6}$ Peter Schellongowski, ${ }^{7}$ \\ David Thickett, ${ }^{2,8}$ Patrick Meybohm, ${ }^{3,9}$ the VITDALIZE Collaboration Group
}

To cite: Amrein K, Parekh D, Westphal S, et al. Effect of high-dose vitamin D3 on 28-day mortality in adult critically ill patients with severe vitamin $D$ deficiency: a study protocol of a multicentre, placebo-controlled double-blind phase III RCT (the VITDALIZE study). BMJ Open 2019;9:e031083. doi:10.1136/ bmjopen-2019-031083

- Prepublication history and additional material for this paper are available online. To view these files, please visit the journal online (http://dx.doi. org/10.1136/bmjopen-2019031083).

KA and DP are joint first authors. DT and PM are joint senior authors.

Received 15 April 2019

Revised 20 August 2019

Accepted 18 September 2019

Check for updates

(c) Author(s) (or their employer(s)) 2019. Re-use permitted under CC BY-NC. No commercial re-use. See rights and permissions. Published by BMJ.

For numbered affiliations see end of article.

Correspondence to Dr Patrick Meybohm; patrick.meybohm@kgu.de

\section{ABSTRACT}

Introduction Observational studies have demonstrated an association between vitamin $\mathrm{D}$ deficiency and increased risk of morbidity and mortality in critically ill patients. Cohort studies and pilot trials have suggested promising beneficial effects of vitamin $D$ replacement in the critical ill, at least in patients with severe vitamin D deficiency. As vitamin $\mathrm{D}$ is a simple, low-cost and safe intervention, it has potential to improve survival in critically ill patients. Methods and analysis In this randomised, placebocontrolled, double-blind, multicentre, international trial, 2400 adult patients with severe vitamin D deficiency (25-hydroxyvitamin $\mathrm{D} \leq 12 \mathrm{ng} / \mathrm{mL}$ ) will be randomised in a 1:1 ratio by www.randomizer.at to receive a loading dose of $540000 \mathrm{IU}$ cholecalciferol within 72 hours after intensive care unit (ICU) admission, followed by 4000 IU daily for 90 days or placebo. Hypercalcaemia may occur as a side effect, but is monitored by regular checks of the calcium level. The primary outcome is all-cause mortality at 28 days after randomisation. Secondary outcomes are: ICU, hospital, 90-day and 1-year mortality; hospital and ICU length of stay, change in organ dysfunction on day 5 as measured by Sequential Organ Function Assessment (SOFA) score, number of organ failures; hospital and ICU readmission until day 90; discharge destination, selfreported infections requiring antibiotics until day 90 and health-related quality of life. Recruitment status is ongoing. Ethics and dissemination National ethical approval was obtained by the Ethics Committee of the University of Graz for Austria, Erasme University Brussels (Belgium) and University Hospital Frankfurt (Germany), and will further be gained according to individual national processes. On completion, results will be published in a peer-reviewed scientific journal. The study findings will be presented at national and international meetings with abstracts online. Trial registration NCT03188796, EudraCT-No: 2016002460-13.

\section{Strengths and limitations of this study}

The VITDALIZE trial is a large, randomised, doubleblind, placebo-controlled, international and multicentre trial.

- The trial will provide important information about efficacy of high dose vitamin $D$ administration in critically ill patients with severe vitamin D deficiency and could be a simple, inexpensive intervention to improve patient outcomes and quality of life.

- Challenges of the trial include the heterogeneous intensive care unit patient cohort and the high number of patients that takes the risk of recruitment failure.

- Due to the differing national processes in consenting and monitoring there may be some variance and each country will abide by their local ethics committee consenting procedures.

\section{INTRODUCTION}

Vitamin D has much broader effects on various metabolic activities than originally expected. ${ }^{1-3}$ Many recent papers have demonstrated the pleiotropic effects of vitamin D. Vitamin D is a precursor of a steroid hormone with a specific nuclear receptor (vitamin D receptor), which regulates more than 1000 genes and is also an important regulator of the immune system. ${ }^{4}$ Besides regulating calcium homeostasis, vitamin D has an effect on muscles, blood vessels, cell proliferation and differentiation, and autoimmune processes. Therefore, vitamin D deficiency causes skeletal and non-skeletal diseases and seems to predispose to a variety of respiratory, 
immune, infectious, neurological and cardiovascular diseases.

25-hydroxyvitamin D $(25(\mathrm{OH}) \mathrm{D})$ is the major circulating vitamin $\mathrm{D}$ metabolite, and its measure best reflects an individual's vitamin D status. Thus, serum $25(\mathrm{OH})$ $\mathrm{D}$ is the generally accepted parameter for determining vitamin $\mathrm{D}$ status. Although the definition for vitamin D deficiency is still under debate, a cut-off of $25(\mathrm{OH})$ $\mathrm{D} \leq 12 \mathrm{ng} / \mathrm{mL} \quad(=30 \mathrm{nmol} / \mathrm{L})$ is uniformly considered to represent severe deficiency. ${ }^{5} 25(\mathrm{OH}) \mathrm{D}$ levels below $12 \mathrm{ng} / \mathrm{mL}$ ( or $30 \mathrm{nmol} / \mathrm{L}$ ) hallmark an increased risk for rickets, osteomalacia and decreased fractional calcium absorption. Although vitamin D supplementation is inexpensive, simple and has an excellent safety profile, testing for and treating of vitamin D deficiency is currently not routinely performed, particularly in the intensive care unit (ICU).

To date, only a few studies have investigated high-dose vitamin $\mathrm{D}$ in critically ill patients with severe vitamin D deficiency. ${ }^{6-8}$ The largest study to date, the VITdAL-ICU randomised, double-blind, placebo-controlled, singlecentre trial included 492 critically ill patients with vitamin $\mathrm{D}$ deficiency $(25(\mathrm{OH}) \mathrm{D} \leq 20 \mathrm{ng} / \mathrm{mL})$ assigned to receive either vitamin D or placebo. ${ }^{6}$ Vitamin D3 or placebo was given orally or via nasogastric tube at a dose of 540000 IU followed by monthly maintenance doses of $90000 \mathrm{IU}$ for 5 months. The study provided no differences between vitamin $\mathrm{D}$ and placebo group concerning the primary outcome of hospital length of stay, hospital mortality or 6-month mortality. However, lower hospital mortality was observed in the severe vitamin $\mathrm{D}$ deficiency subgroup $(25(\mathrm{OH}) \mathrm{D} \leq 12 \mathrm{ng} / \mathrm{mL}, \mathrm{n}=200$ or $42 \%$ of the total population). As this was only a secondary endpoint in the predefined subgroup with severe vitamin D deficiency, this finding is hypothesis generating and prompted the current VITDALIZE study. We hypothesise that vitamin D replacement will improve patient's outcome and quality of life in critically ill patients with severe vitamin D deficiency.

\section{METHODS AND ANALYSIS}

\section{Trial design}

The VITDALIZE trial is a multicentre, randomised, placebo-controlled, double-blind phase III trial targeting a sample size of 2400 critically ill patients with severe vitamin D deficiency in more than 30 sites in Austria, Germany, Belgium, Switzerland and UK.

The aim of the trial is to determine if high-dose vitamin D3 improves clinical outcomes and is cost-effective in comparison to placebo in adult critically ill patients with severe vitamin $\mathrm{D}$ deficiency. An extended version of the protocol is added as an online supplementary file (Protocol_24.01.2018-1.3).

\section{Trial population}

The trial population consists of mixed adult critically ill patients screened within 72 hours after ICU admission anticipated to require $\geq 48$ hours of ICU care at the time of screening with documented vitamin $\mathrm{D}$ deficiency using local routine testing $(25(\mathrm{OH}) \mathrm{D} \leq 12 \mathrm{ng} / \mathrm{mL}$ $(=30 \mathrm{nmol} / \mathrm{L}))$ recruited in several countries in academic and non-academic hospitals. In the UK, where it is not possible to test for vitamin D levels in 1 day, samples will be processed by an FDA (Food and Drug Administration)/CE (Conformité Européenne) approved rapid bedside vitamin D3 analyser (Sofia, Quidel, USA) that will determine if the patient has severe vitamin $\mathrm{D}$ deficiency $(25(\mathrm{OH}) \mathrm{D} \leq 12 \mathrm{ng} / \mathrm{mL})$.

A flowchart of study intervention is seen in figure 1 and a schedule of assessment and procedures in table 1.

The day of the study medication loading dose is day 0 (V1). At day 5 (V2), extensive clinical data will be collected. The primary endpoint (28-day mortality) will be assessed at V3. A follow-up visit will be done by telephone 90 days after randomisation with patient or family physician. This visit will include important safety evaluations and secondary outcomes. A final follow-up visit will be done after 1 year.

\section{Recruitment}

The single-centre pilot study (VITdAL-ICU) was conducted at the Medical University of Graz, a large tertiary academic centre with 123 ICU beds. During a 2-year period, we were able to recruit $n=492$ critically ill adult patients with vitamin $\mathrm{D}$ deficiency $(<20 \mathrm{ng} / \mathrm{mL})$. Severe vitamin D deficiency was found in $42 \%$, or 200 patients. ${ }^{6}$ Current data from recruiting sites indicate that severe vitamin D deficiency in critically ill patients is between $30 \%$ and $50 \%$. We will need to recruit an average of approximately 2-3 patients per month. Recruitment rates are based on previous ICU trials, the VITdAL-ICU study and an accepted screen failure rate of 2 out of 3 for severe vitamin D deficiency. Given the high prevalence of severe vitamin D deficiency and broad eligibility criteria we believe this is a conservative but realistic recruitment target.

The per-site recruitment will be monitored actively at nationally coordinated trial management group meetings. We will aim to identify barriers and solutions to help improve the recruitment. Advice from sites with a high level of recruitment will be disseminated via teleconference. Advice on how to maximise recruitment will be sought from our patient partners on a regular basis. If centres consistently fail to meet recruitment targets, we will recruit additional sites while minimising the resources used in maintaining poorly recruiting sites or allow higher recruitment rates than planned in highrecruiting centres.

\section{Randomisation and blinding}

Randomisation and blinding will be performed using the secure and validated web-based randomisation service 'Randomizer for Clinical Trials' (Institute for Medical Informatics, Statistics and Documentation, Medical University of Graz, Austria; available at:www.randomizer. 
Critically ill patients in ICU

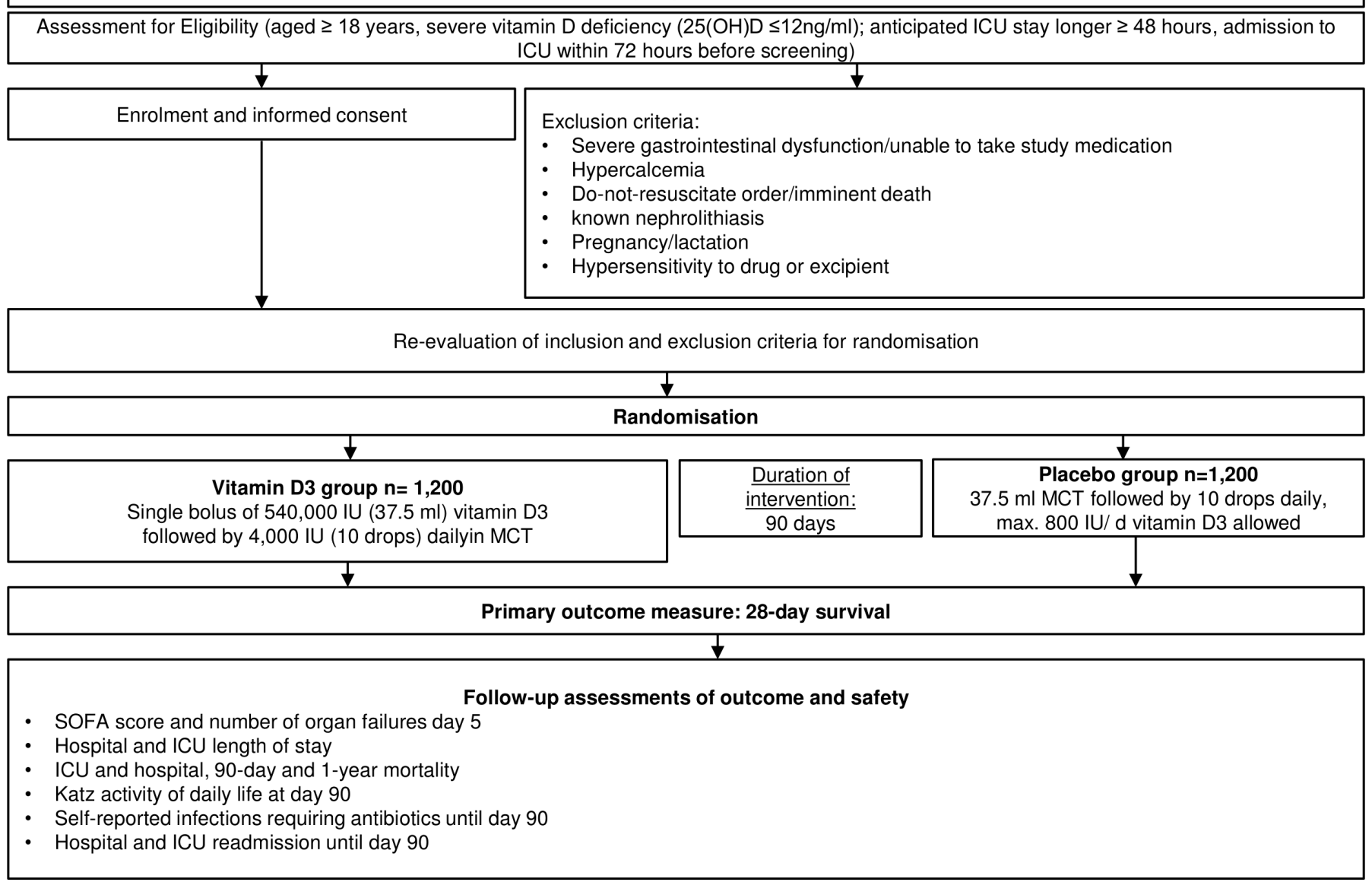

Final assessment: Survival status at 12 months

Figure 1 Trial flow of intervention scheme. We will check eligibility and obtain informed consent* from patients or legally authorised representative/healthcare proxy. After evaluation of exclusion criteria, patients will be randomised in the intervention or placebo group. Primary endpoint is 28-day all-cause mortality. *When informed consent is not possible at time of screening, country-specific alternative strategies for obtaining informed consent are used (ie, in Austria delayed informed consent, in Germany, consent of legal representative, England and Wales consent of relatives or responsible clinician). ICU, intensive care unit; IU, international unit; MCT, median chain triglycerides; SOFA, Sequential Organ Failure Assessment.

at). Patients will be randomly assigned to either vitamin D or placebo in a 1:1 ratio and stratified according to centre and gender. To ensure equal group sizes a restricted randomisation method will be used.

Allocation concealment will be ensured, as the service will not release the randomisation code until the patient has been recruited into the trial.

The following method will be used to maintain blinding: the blinding list will be kept strictly confidential and no routine vitamin $\mathrm{D}$ testing is done after study inclusion. An independent statistician and unblinded pharmacist will keep treatment allocation information confidential until database lock. Patient's group allocation will not be revealed until final statistical analysis. In case of safety concerns (eg, severe hypercalcaemia $>3.5 \mathrm{mmol} / \mathrm{L}$ ), participants of the study may be unblinded by the local investigator at each participating site and/or the coordinating centre. This will be done and documented with the Randomizer.

\section{Intervention}

- Vitamin D3 group receiving a bolus of 540000 I.U. vitamin D3, dissolved in medium chain triglycerides (MCT, 37.5 mL), at day 0 followed by 4000 IU daily (10 drops) for 90 days, or

- Placebo group receiving $37.5 \mathrm{~mL}$ MCT solution at day 0 followed by 10 drops MCT for 90 days.

A concomitant routine low dose vitamin D intake of up to 800 IU daily is permitted, but very unlikely to have an effect in this 90-day time frame and population.

\section{Inclusion and exclusion criteria}

Inclusion criteria

- Age $\geq 18$ years.

- Anticipated ICU stay $\geq 48$ hours.

- Admission to ICU $\leq 72$ hours before screening.

- Severe vitamin D deficiency $(\leq 12 \mathrm{ng} / \mathrm{mL}$ $(30 \mathrm{nmol} / \mathrm{L})$ or undetectable) using local routine testing. 
Table 1 Frequency and scope of study visits

\begin{tabular}{|c|c|c|c|c|c|c|}
\hline & & $\begin{array}{l}\text { Enrolment, } \\
\text { baseline data (V1) }\end{array}$ & $\begin{array}{l}\text { Clinical data } \\
\text { (V2) }\end{array}$ & $\begin{array}{l}\text { 28-day } \\
\text { mortality (V3) }\end{array}$ & $\begin{array}{l}\text { 90-day follow- } \\
\text { up (V4) }\end{array}$ & $\begin{array}{l}\text { 1-year follow- } \\
\text { up (V5) }\end{array}$ \\
\hline Assessment & Screening (V0) & Day 0 & Day 5 & Day 28 & Day 90 & Month 12 \\
\hline Informed consent & & $\mathrm{X}$ & & & & \\
\hline Demographics & & $x$ & & & & \\
\hline \multicolumn{7}{|l|}{ Intervention } \\
\hline $\begin{array}{l}\text { Loading dose } 540000 \\
\text { IU } \\
\text { vitamin D3 }\end{array}$ & & $x$ & & & & \\
\hline $\begin{array}{l}\text { Daily dose } 4000 \text { IU } \\
\text { vitamin D3 }\end{array}$ & & & $x$ & $x$ & $x$ & \\
\hline $\begin{array}{l}\text { Infections requiring } \\
\text { antibiotics }\end{array}$ & & & & & $x$ & \\
\hline $\begin{array}{l}\text { Hospital and ICU } \\
\text { readmission }\end{array}$ & & & & & $x$ & \\
\hline Katz activity of daily life & & $x$ & & & $\mathrm{X}$ & \\
\hline \multicolumn{7}{|l|}{ Safety evaluation } \\
\hline Serum calcium & $x$ & $x$ & $x$ & & & \\
\hline Falls/fractures & & & & & $\mathrm{X}$ & \\
\hline $\begin{array}{l}\text { New episodes of } \\
\text { nephrolithiasis }\end{array}$ & & & & & $x$ & \\
\hline
\end{tabular}

ICU, intensive care unit; SOFA, Sequential Organ Function Assessment.

\section{Exclusion criteria}

- Severe gastrointestinal dysfunction/unable to receive study medication.

- Patients with a do not resuscitate order/imminent death.

- Not expected to survive initial 48 hours of admission or treatment withdrawal imminent within 24 hours.

- Hypercalcaemia ( $>2.65 \mathrm{mmol} / \mathrm{L}$ total calcium and/or $>1.35 \mathrm{mmol} / \mathrm{L}$ ionised calcium at screening).

- Known kidney stones, active tuberculosis or sarcoidosis (within the last 12 months).

- Pregnancy/lactation.

- Hypersensitivity to drug or excipient.

\section{Outcome measures}

The primary outcome of this trial will be all-cause mortality at day 28 after randomisation.

Secondary outcomes include 90-day and 1-year allcause mortality, ICU and hospital mortality and length of stay, change in organ dysfunction on day 5 as measured by the SOFA score (Sequential Organ Function Assessment) and the number of organ failures $(0-6$; as defined by $>2$ SOFA points in each of the six categories). The six categories comprise the respiratory system $\left(\mathrm{PaO}_{2} / \mathrm{FiO}_{2}\right)$, the nervous system (Glasgow coma scale), the cardiovascular system (mean arterial pressure OR administration of vasopressors required), the liver (bilirubin), the coagulation (number of platelets) and the kidney (creatinine or urine output).

Further secondary endpoints are hospital and ICU readmission rate until day 90, discharge destination (home, rehabilitation, other hospital), self-reported infections requiring antibiotics until day 90 and the Katz activities of daily life, the most appropriate instrument to assess functional status as a measurement of the patient's ability to perform basic activities independently, at day 90 will be collected using a questionnaire.

Mortality at day 28, day 90 and 1 year will be enquired by telephone, through hospital information system and national data linkage systems (where available). SOFA scores will be generated by collection of routinely collected clinical data.

Safety outcomes comprise hypercalcaemia at day 5 , selfreported or documented falls and fractures until day 90 and new episodes of kidney stones.

In the UK arm, a near patient testing device for rapid vitamin $\mathrm{d}$ assessment will be used. Additional secondary outcomes are Health-related quality of life (EQ-5D-5L, a standardised instrument for measuring generic health 
status) and a disability assessment (WHO-DAS 2.0 (WHO Disability Assessment Schedule 2.0), a generic assessment instrument for health and ability) at 90 days and 1 year. EQ-5D-5L and WHODAS 2.0 EQ-5D are designed for selfcompletion and as such captures information directly from the respondent. Further additional secondary endpoints in the UK arm are secondary healthcare utilisation in the first year (ICU and hospital length of stay, readmissions and utilisation of hospital and community care resources after hospital discharge 1 year after randomisation), from Hospital Episode Statistics, civil registry data held by NHS Digital and patient questionnaires and health economics analysis including cost effectiveness of screening for and treating vitamin D deficiency in critical illness and cost per quality-adjusted life year gained 1 year after randomisation and at end of life.

\section{Monitoring}

Patients will be monitored daily until ICU discharge and serious adverse events will be reported to the regulatory authorities, other participating centres and the manufacturer of the study medication. Following site-initiation the need for further site monitoring visits will be assessed on an individual and risk proportionate approach. Further site monitoring visits may be triggered by the following: poor quality data returns, repeated issues with randomisation (ineligible patients being entered), repeated issues with consenting, non-compliance with the protocol or good clinical practice, unusual data patterns or safety reporting issues. This may vary depending on national oversight processes.

The DSMB (data safety monitoring board) is responsible for safeguarding the interests of trial subjects, assessing the safety and efficacy of the interventions during the trial, and for monitoring the overall conduct of the clinical trial. The DSMB will assess the progress of the trial at regular intervals ( $\sim 6$ months) and will evaluate all safety data. In addition, the DSMB will evaluate the results of the interim efficacy analysis. It will recommend to the coordinating investigator and the sponsor whether to continue or stop the trial.

\section{Sample size considerations}

The sample size for this multinational study is based on the primary endpoint 28-day mortality. In the VITdAL-ICU study, 28-day mortality rates of $36 \%(37 / 102)$ in the placebo group and 20\% (20/98) in the vitamin D Group were observed. ${ }^{6}$ The VITDALIZE study has been designed to be sufficiently powered to detect a smaller, but clinically relevant absolute mortality difference of $5 \%$ with a power of $80 \%$ with an assumed baseline mortality rate of $25 \%$. This corresponds to a clinically highly relevant relative risk reduction of $20 \%$.

Multicentre trials generally have a smaller treatment effect than monocentre studies. ${ }^{9}$ We assume that this will also be the case for the VITDALIZE study. Furthermore, our assumed 5\% absolute mortality difference is in line with a recent survey among clinical intensivists that the largest median treatment effect considered plausible by intensivists for current ongoing ICU multicentre trials is $3 \%-5 \% .^{10}$

Using a fixed sample size design and a two-sided logrank test for equality of survival curves with a two-sided alpha level of $5 \%$, a sample size of $n=1093$ per group will be needed to achieve a power of $80 \%$ (total sample size of 2186).

Incorporating one interim analysis after inclusion of $50 \%$ of the patients a total sample size of at least $\mathrm{n}=2194$ (494 events) is required to achieve $80 \%$ power using an O'Brien-Fleming spending function. ${ }^{11}$ Accounting for a drop-out rate of approximately $10 \%$ yields a total sample size of $n=2400$ patients. For sample size calculation the software package nQuery 7.0+nTerim 2.0 was used.

\section{Statistical analysis}

The primary analysis will be performed on the intentionto-treat population (ITT). The ITT will include all patients who receive at least the loading dose of the study medication. All patients will be analysed according to the treatment assignment during randomisation. The per protocol population will include all patients who received the loading dose and have a compliance $>80 \%$. Compliance is defined as self-reported percentage of doses ingested until day 90 .

The safety analyses will be based on the treated set, which is defined as all randomised patients who receive at least one dose of trial medication. All patients will be analysed according to the treatment they received.

\section{Data analysis}

All clinical and safety data collected in the study will be analysed with SAS V.9.4. Data will be presented as summary tables and, where appropriate, as plots. Continuous data will be described by means, standard deviation, medians and upper and lower quartiles unless otherwise stated. The number of observations and minimum and maximum values are also included.

Categorical data will be summarised using frequencies and percentages.

The primary outcome, 28-day all-cause mortality, will be displayed using Kaplan-Meier estimates of survival curves in each treatment arm. Group comparison will be made using a stratified two-sided log rank test. The unstratified log-rank test will be performed as a sensitivity analysis. The Cox regression model, including treatment and stratification factor, will be used to estimate the hazard ration and its $95 \%$ confidence interval. Details will be defined in a Statistical Analysis Plan.

ICU, hospital mortality, 90-day mortality and 1-year mortality will be analysed as secondary outcomes using Kaplan-Meier estimates of survival curves. For the other secondary parameters, comparison between groups will be performed using appropriate parametric or nonparametric methods and $\chi^{2}$ tests.

The safety outcomes, (hypercalcaemia on day 5, new kidney stones, self-reported falls and fractures until day 
90) will be analysed as binary variables and compared with $\chi^{2}$ tests.

\section{Demographic and baseline characteristics}

In a summary of demographic, baseline and diagnostic characteristics (age, weight, height, sex, SAPS III, Charlson comorbidity index) a comparison of the treatment groups will be performed. To this end, appropriate descriptive and inferential statistics will be applied. Relevant medical history will be also displayed using summary statistics according to the two treatment groups.

\section{Subgroup analysis}

Predefined subgroup analyses will be performed for all primary and secondary outcomes based on the following group definitions as exploratory analysis:

Kidney function (CKD 4 or lower vs higher at inclusion). Sepsis (admission diagnosis) versus non-sepsis defined by the 2016 criteria (suspected infection/qSOFA on day 0 -respiratory rate of $22 / \mathrm{min}$ or greater, altered mentation, or systolic blood pressure of $100 \mathrm{~mm} \mathrm{Hg}$ or less).

\section{Missing data handling}

All available data will be used in the analyses and data summaries. There will be no imputation of any missing data.

\section{Planned interim analysis}

The trial uses a group sequential design with one interim analysis when $50 \%$ of the planned enrolled patients in each arm ( $\mathrm{n}=600$ per arm) have completed their day 28 assessment by the independent data safety monitoring board. The enrolment of patients will continue while the interim analysis is performed. This interim analysis is intended to test for efficacy, that is, the trial may be terminated after the interim analysis, if the main question can already be answered at this interim analysis. If the interim analysis shows a benefit for the vitamin D group, the DSMB may recommend early study termination. The interim analysis will be performed only for the primary outcome 28-day mortality. The O' Brien-Fleming rule will be used to stop the trial early for efficacy. In detail, if the $\mathrm{p}$ value of the log rank test is smaller than 0.003 , then the trial can be stopped early by the DSMB.

\section{DISCUSSION}

Recent studies have demonstrated that low vitamin D levels are an independent risk factor for mortality in critically ill patients ${ }^{12-17}$ reflecting the relevant role of vitamin D.

Worldwide, the prevalence of vitamin $\mathrm{D}$ deficiency in intensive care patients ranges between $40 \%$ and $70 \%$. Therapeutic interventions like surgery, fluid resuscitation, extracorporeal membrane oxygenation, cardiopulmonary bypass, dialysis and plasma exchange and hepatic, parathyroid and renal dysfunction may significantly reduce vitamin D levels. ${ }^{18}$

Pleiotropic effects of vitamin D on the immune system, glucose metabolism, and calcium homeostasis are essential in critically ill patients. Vitamin D deficiency carries an additional risk due to mortality and morbidity to these patients. ICU patients often suffer from immunological dysfunction and changes in body composition (loss of muscle mass, increase in the adipose tissue). Every additional day staying on ICU increases the chance of becoming dependent on care with prolonged rehabilitation and recovery time. Interventions, such as vitamin D supplementation, may also have the potential to improve health related quality of life.

A recent Cochrane meta-analysis ${ }^{19}$ with 50623 adults who were healthy or were recruited among the general population, or diagnosed with a specific disease, showed that vitamin D3 supplementation was linked to significantly improved survival. An individual patient data analysis from eight major vitamin D trials with $>70000$ participants showed a reduction of mortality by vitamin D by $9 \% .^{20}$ Genetically low $25(\mathrm{OH}) \mathrm{D}$ is associated with increased all-cause mortality. ${ }^{21}$ An observational cohort study of 4344 adults hospitalised between 1993 and 2011 demonstrated that in those patients with prehospital $25(\mathrm{OH}) \mathrm{D}$ concentrations $<20 \mathrm{ng} / \mathrm{mL}$, an improvement in vitamin D status during the year leading up to hospitalisation was independently associated with improved allcause mortality and decreased hospital length of stay. ${ }^{22}$

Recently, in a double-blind, randomised controlled study, it was demonstrated that the administration of high dose vitamin D (up to $500000 \mathrm{IU}$ ) increased levels of antimicrobial molecules ${ }^{23}$ that may have beneficial effects on critical illness and inflammatory outcomes.

Although vitamin D supplementation is inexpensive, simple and has an excellent safety profile, testing for and treating of vitamin $\mathrm{D}$ deficiency is currently not routinely performed in ICU patients.

The VITDALIZE trial is a large randomised, multicentre international study designed to demonstrate the clinical benefit of vitamin D supplementation in critically ill patients with severe vitamin $\mathrm{D}$ deficiency. The primary outcome will be 28-day all-cause mortality. All-cause mortality represents a 'hard' endpoint that is not prone to measurements bias. Most importantly, the European Medicines Agency recently recommended short-term (28 day) all-cause mortality as the most relevant primary efficacy endpoint in confirmatory clinical trials assessing the efficacy of drugs or medicinal products in patients with life-threatening acute illnesses, for example, in critically ill patients with sepsis ${ }^{24}$ or with acute respiratory distress syndrome.$^{25}$ Long-term effects will be reflected by the secondary endpoints 90 days and 1-year mortality and the secondary endpoint Katz Activity of Life will reflect the health related quality of life. The UK arm will also be assessing the cost-effectiveness and health economics with further health-related quality measures of the intervention.

Due to the differing national processes in consenting and monitoring there may be some variance and each country will abide by their local ethics committee consenting procedures. 
The VIOLET (Vitamin D to improve outcomes by leveraging early treatment; ClinicalTrials.gov Identifier: NCT03096314) trial is another important and similar, yet substantially different RCT that has stopped recruitment in July 2018 but no results have been published at the time of writing. VIOLET included patients with vitamin $\mathrm{D}$ deficiency (point-of-care test, $<20 \mathrm{ng} / \mathrm{mL}$ ), but not necessarily ICU patients. The intervention consisted of a single bolus loading dose (540000 IU vitamin D3), but no maintenance dose; and the primary endpoint is 90 -day mortality. Together, these two large trials in acutely ill patients will greatly advance our knowledge in this field.

As vitamin D3 application is a simple, low-cost, safe and well-tolerated intervention, it has great potential to improve survival and quality of life in critically ill patients and could be implemented worldwide immediately.

\section{ETHICS AND DISSEMINATION}

Study protocol (V.1.3, EudraCT-No. 2016-00246013), patient information, and informed consent were approved by the leading Ethics Committee of the University of Graz (EK 1289/2016), and will be submitted to each participating trial centre. In the meanwhile, study protocol has been approved by the leading national Ethics Committee of the Erasme University Brussels for Belgium and University Hospital Frankfurt for Germany.

Informed consent to participate in the study will be gained by patients, relatives, physicians, and/or legal represenatives, depending on national regulatory specifications.

Recruitment in Austria started in October 2017 and in Belgium in January 2019. The current study protocol (V.1.3) was released in January 2018. This trial was registered at http:/ / clinicaltrials.gov in June 2017. So far, more than 390 patients have been randomised in $>15$ active centres. The planned recruitment lasts for approximately another 36 months. The UK is funded by NIHR HTA and projected to start recruitment in 2020. Germany will be funded by the BMBF (Federal Ministry of Education and Research) and will start recruitment in 2020. In addition, ESICM and Fresenius Kabi support funding. The participation of Switzerland is planned, but will depend on funding possibilities.

On completion, results will be published in a peerreviewed scientific journal.

\footnotetext{
Author affiliations

${ }^{1}$ Department of Internal Medicine, Medical University of Graz, Graz, Austria ${ }^{2}$ Birmingham Acute Care Research Group, University Hospitals Birmingham NHS Foundation Trust, Birmingham, UK

${ }^{3}$ Department of Anesthesiology, Intensive Care Medicine and Pain Therapy, University Hospital Frankfurt, Goethe University, Frankfurt, Germany ${ }^{4}$ Intensive Care Medicine, Erasme University Hospital, Brussels, Belgium ${ }^{5}$ Institute for Medical Informatics, Statstics, and Documentation, Medical University of Graz, Graz, Austria

${ }^{6}$ Intensive Care Unit, Department of Internal Medicine, University Hospital of Graz, Graz, Austria

${ }^{7}$ University Hospital of Internal Medicine I, Medical University Wien, Wien, Austria
}

${ }^{8}$ University of Birmingham School of Clinical and Experimental Medicine, Birmingham, UK

${ }^{9}$ Department of Anesthesiology, University Hospital Wuerzburg, Wuerzburg, Germany

Twitter Dhruv Parekh @drdhruvparekh

Collaborators The VITDALIZE collaboration group will be listed as an author, and the names of the individual members of the group will be searchable through their individual PubMed records: AUSTRIA: Barmherzige Brueder, Vienna, Austria: Corinna Marschalek (corinna.marschalek@bbwiwn.at), Rene Schmutz (rene.schmutz@ bbwien.at); Barmherzige Brueder, Linz, Austria: Martin Clodi (martin.clodi@bblinz. at), Michael Resl (michael.res।@bblinz.at); Ordensklinikum Linz, Austria: Mario Krasser (mario.krasser@ordensklinikum.at), Matthias Michlmayr (matthias. michlmayr@ordensklinikum.at), Johann Reisinger (johann.reisinger@ ordensklinikum.at), Alexandra Schiller (alexandra.schiller@ordensklinikum.at); Medical University Graz, Austria: Nicolas Dominik Verheyen (nicolas.verheyen@ medunigraz.at), Dirk von Lewinski (dirk.von-lewinski@medunigraz.at), Marlene Sandra Deininger (marlene.deininger@medunigraz.at), Andreas Muench (andreas. muench@medunigraz.at), Holger Simonis (holger.simonis@medunigraz.at), Paul Zajic (paul.zajic@medunigraz.at), Ines Lindenau (ines.lindenau@medunigraz.at), Philipp Eller (Philipp.eller@medunigraz.at), Alexander Holl (alexander.holl@ klinikum-graz.at), Alexander Pichler (alexander.pichler@medunigraz.at), Tadeja Urbanic Purkart (tadeja.urbanic-purkart@medunigraz.at), Magdalena Hoffmann ( Magdalena.hoffmann@medunigraz.at), Katharina Ritsch (katharina.ritsch@ medunigraz.at), Simon Schmidt (simon.schmidt@medunigraz.at); Medical University Innsbruck, Austria: Romuald Bellmann (romuald.bellmann@tirol-kliniken. at), Anna Brandtner (anna.brandtner@tirol-kliniken.at), Adelheid Ditlbacher ( adelheid.ditlbacher@tirol-kliniken.at), Julia Hasslacher (julia.hasslachen@tirolkliniken.at), Michael Joannidis (michael.joannidis@i-med.ac.at), Andreas Peer (a. peer@tirol-kliniken.at), Klemens Zotter (klemens.zotter@tirol-kliniken.at); Kardinal Schwarzenberg Klinikum, Schwarzach, Austria: Christoph Martin Biedermann ( christoph.biedermann@ks-klinikum.at), Andreas Valentin (andreas.valentin@ksklinikum.at), Franz Wimmer (franz.wimmer@ks-klinikum.at); Klinikum Klagenfurt am Wörthersee, Klagenfurt, Austria: Susanne Demschar (susanne.demschar@ kabeg.at), Markus Koestenberger (markus.koestenberger@kabeg.at), Rudolf Likar ( rudolf.likar@kabeg.at), Stefan Neuwersch (stefan.neuwersch@kabeg.at), Michael Pogatschnigg (michael.pogatschnigg@kabeg.at), Brigitte Trummer (brigitte. trummer@kabeg.at); Kepler University Hospital, Linz, Austria: Martin Duenser ( martin.duenser@keplerklinikum.at), Jens Meier (jens.meier@kepleruniklinikum.at); LKH Leoben, Standort Bruck/ Mur, Austria: Johann Kainz (johann.kainz@kages.at), Romana Sommer (romana.sommer@kages.at); LKH Graz II, Standort Enzenbach, Austria: Otmar Schindler (otmar.schindler@kages.at); LKH Feldbach, Austria: Alexander Lehr (alexander.lehr@kages.at), Guenter Mesaric (guenter.mesaric@ kages.at), Yasmin Tinawi (yasmin.tinawi2@kages.at), Norbert Watzinger (norbert. watzinger@kages.at); LKH Fuerstenfeld, Austria: Randolf Hammerl (randolf. hammerl@kages.at), Peter Krippl (peter.krippl@kages.at), Stefan Langner (Stefan. langner@kages.at), Martin Lux (martin.lux@kages.at), Matthias Unteregger ( matthias.unteregger@kages.at); LKH Hochsteiermark, Standort Leoben, Austria: Gerwig Fruehauf (gerwig.fruehauf@kages.at), Barbara Gruber (barbara.gruber@ kages.at), Bertram Harzl (bertram.harzl@|kh-leoben.at), Viktor Wutzl (viktor.wutz।@ kages.at); Barmherzige Brueder St.Veit/ Glan, Austria: Juergen Berghofer (juergen. berghofer@bbstveit.at), Nadine Fuch (nadine.fuchs@bbstveit.at), Gilbert Hainzl ( gilbert.hainzl@bbstveit.at), Isolde Pessentheiner (Isolde.pessentheiner@bbst.veit. at), Michael Zink (michael.zink@bbstveit.at); LKH Villach, Austria: Petra Hoernler ( petra.hoernler@kabeg.at), Ernst Trampitsch (ernst.trampitsch@kabeg.at); Medical University Vienna, Austria: Jakob Muehlbacher (jakob.muehlbacher@meduniwien. ac.at), Philipp Riss (philipp.riss@meduniwien.ac.at), Roman Ullrich (roman.ullrich@ meduniwien.ac.at), Martin Bernardi (martin.bernardi@meduniwien.ac.at), Michael Hiesmayr (michael.hiesmayr@meduniwien.ac.at), Katharina Kovac (katharina. kovac@meduniwien.ac.at), Cecilia Veraar (cecilia.veraar@meduniwien.ac.at), Bernhard Zapletal (bernhard.zapletal@meduniwien.ac.at), Elisabeth Lobmeyr ( elisabeth.Iohmeyr@meduniwien.ac.at), Esther Tiller (estehr.tiller@meduniwien.ac. at); Wiener Krankenanstaltverband, Vienna, Austria: Georg Hinterholzer (georg. hinterholzer@wienkav.at), Daniel Mydza (daniel.mydza@wienkav.at), Sabine Schmaldienst (sabine.schmaldienst@wienkav.at). BELGIUM: CHU de Charleroi, Belgium: Maxime van Cutsem (maxime.vancutsem@chu-charleroi.be), CHU Ambroise Paré, Mons, Belgium: Marianne Blockmans (marianne.blockmans@hap. be), Alain D'hondt (alain.dhondt@hap.be); CHR Citadelle, Liège, Belgium: Vincent Fraipont (vincent.fraipont@chrcitadelle.be), Sophie Jacquet (sophie.jacquet@ chrcitadelle.be); Erasme University Hospital, Brussels, Belgium: Caroline Abbenhuijs (caroline.abbenhuijj@erasme.ulb.ac.be); Katarina Halenarova (katarina. halenarova@erasme.ulb.ac.be), Romain Courcelle (romain.courcelle@erasme.ulb. 
ac.be), Dominique Durand (dominique.durand@erasme.ulb.ac.be), Amedee Ego ( amedee.ego@erasme.ulb.ac.be), Amina Khaldi (amina.khaldi@erasme.ulb.ac.be), Itesa Nancy Matumikina (itesa.nancy.matumikina@erasme.ulb.ac.be). GERMANY: University Hospital Frankfurt, Germany: Kai Zacharowski (kai.zacharowski@kgu.de), Simone Lindau (simone.lindau@kgu.de), Philipp Helmer (philipp.helmer@kgu.de); University Hospital Schleswig-Holstein, Campus Kiel, Germany: Gunnar Elke ( gunnar.elke@uksh.de), Norbert Weiler (norbert.weiler@uksh.de), Lars Hummitzsch ( lars.hummitzsch@uksh.de), Guenther Zick (guenther.zick@uksh.de), Tobias Becher (tobias.becher@uksh.de), Dirk Schaedler (dirk.schaedler@uksh.de), Matthias Kott ( matthias.kott@uksh.de), Barbara Vogt (barbara.vogt@uksh.de), Ingmar Lautenschlaeger (Ingmar.lautenschlaeger@uksh.de); University Hospital SchleswigHolstein, Campus Luebeck, Germany: Georg Fuernau (georg.fuernau@uksh.de); University Hospital Bonn: Christian Putensen (Christian.putensen@ukb.uni-bonn. de), Stefan Ehrentraut (stefan.ehrentraut@ukb.uni-bonn.de), Maria Wittmann ( maria.wittmann@ukbonn.de); Klinikum rechts der Isar, TUM, Munich, Germany: Barbara Kapfer (b.kapfer@tum.de); Muenchen Klinikum Neuperlach, Munich, Germany: Thomas Felbinger (thomas.felbinger@muenchen-klinik.de), Ines Kaufmann (ines.kaufmann@muenchen-klinik.de); HGZ Bad Bevensen, Germany: Bjoern Andrew Rempiss (ba.rempiss@hgz-bb.de); Charité-Universitaetsmedizin: Claudia Spiess (Claudia.spiess@charite.de), Stefan Schaller (stefan.schaller@ charite.de); University Hospital Wuerzburg, Wuerzburg, Germany: Patrick Meybohm ( meybohm_p@ukw.de), Peter Kranke (kranke_p@ukw.de), Tobias Schlesinger ( schlesinge_t@ukw.de), Markus Kredel (kredel_m@ukw.de), Magdalena Sitter ( sitter_m@ukw.de), Jan Stumpner (stumpner_j@ukw.de), Nico Schlegel (schlegel_ n@ukw.de), Christoph Schimmer (schimmer_c@ukw.de), Ekkehard Kunze (kunze_ e@ukw.de); Universitätsmedizin Mainz, Mainz, Germany: Thomas Kerz (thomas. kerz@unimedizin-mainz.de), Dominik Wesp (dominik.wesp@unimedizin-mainz.de), Axel Neulen (axel.neulen@unimedizin-mainz.de), Florian Ringel (florian.ringel@ unimedizin-mainz.de); RWTH Aachen University Hospital, Aachen, Germany: Christian Stoppe (christian.stoppe@gmail.com); SWITZERLAND: University Hospital Zurich, Switzerland: Mario Maggiorini (mario.maggiorini@usz.ch); Bern University Hospital, Switzerland: Stephan Jakob (stephan.jakob@insel.ch), Joerg Schefold ( joerg.schefold@insel.ch).UK: BCTU, Birmingham, UK: Rebekah Wale, (r.wale@bham. ac.uk); Gemma Slinn, (g.slinn@bham.ac.uk); Peter Brocklehurst, (p.brocklehurst@ bham.ac.uk); Louise Jackson, (l.jackson.1@bham.ac.uk); Queen's University Belfast, UK: Danny McAuley (d.f.mcauley@ qub.ac.uk); UHB, Birmingham, UK: Teresa Melody (teresa.melody@heartofengland.nhs.uk); Jaimin Patel (jaimin.patel@ uhb.nhs.uk); Warwick University, UK: Gavin Perkins (g.d.perkins@warwick.ac.uk); GSTT, London, UK: Marlies 0stermann (marlies.ostermann@gstt.nhs.uk); Cardiff University, UK: Matthew Morgan (morganmjw@ cardiff.ac.uk); UCLH, London, UK: Mervyn Singer (msinger@ ucl.ac.uk); University of Oxford, UK: Matthew Rowland (matthew.rowland@ndcn.ox.ac.uk)

Contributors All authors have substantially contributed to the interpretation of current specific knowledge, which resulted in the conception and design of the present trial. KA, DP, SW, JCP, PE, PS, DT and PM are investigators of the present trial and participated in the acquisition of funding and contribute to the collection of data. $A B$ and RR wrote the statistical methods. All authors have been involved in drafting the manuscript or revising it critically for important intellectual content, and approved the final manuscript.

Funding This work is supported by the Federal Ministry of Education and Research (BMBF 01KG1815), the European Society of Intensive Care Medicine (ESICM), Fresenius Kabi and the National Institute for Health Research (NIHR).

Competing interests None declared.

Patient consent for publication Not required.

Provenance and peer review Not commissioned; externally peer reviewed.

Open access This is an open access article distributed in accordance with the Creative Commons Attribution Non Commercial (CC BY-NC 4.0) license, which permits others to distribute, remix, adapt, build upon this work noncommercially, and license their derivative works on different terms, provided the original work is properly cited, appropriate credit is given, any changes made indicated, and the use is non-commercial. See: http://creativecommons.org/ licenses/by-nc/4.0/.

\section{ORCID iDs}

Dhruv Parekh http://orcid.org/0000-0002-1508-8362

Philipp Eller http://orcid.org/0000-0001-5537-3646
REFERENCES

1 Bikle D. Nonclassic actions of vitamin D. J Clin Endocrinol Metab 2009;94:26-34.

2 Martineau AR, Jolliffe DA, Hooper RL, et al. Vitamin D supplementation to prevent acute respiratory tract infections: systematic review and meta-analysis of individual participant data. BMJ 2017;356.

3 Amrein K, Papinutti A, Mathew E, et al. Vitamin D and critical illness: what endocrinology can learn from intensive care and vice versa. Endocr Connect 2018;7:R304-15.

4 Colotta F, Jansson B, Bonelli F. Modulation of inflammatory and immune responses by vitamin D. J Autoimmun 2017;85:78-97.

5 Ross AC. The 2011 report on dietary reference intakes for calcium and vitamin D. Public Health Nutr 2011;14:938-9.

6 Amrein K, Schnedl C, Holl A, et al. Effect of high-dose vitamin D3 on hospital length of stay in critically ill patients with vitamin D deficiency: the VITdAL-ICU randomized clinical trial. JAMA 2014;312:1520-30.

7 Leclair TR, Zakai N, Bunn JY, et al. Vitamin D supplementation in mechanically ventilated patients in the medical intensive care unit. JPEN J Parenter Enteral Nutr 2019. doi:10.1002/jpen.1520. [Epub ahead of print: 12 Feb 2019].

8 Langlois PL, Szwec C, D'Aragon F, et al. Vitamin D supplementation in the critically ill: a systematic review and meta-analysis. Clin Nutr 2018;37:1238-46.

9 Dechartres A, Boutron I, Trinquart L, et al. Single-center trials show larger treatment effects than multicenter trials: evidence from a metaepidemiologic study. Ann Intern Med 2011;155:39-51.

10 Ridgeon EE, Young PJ, Bellomo R, et al. The fragility index in multicenter randomized controlled critical care trials. Crit Care Med 2016;44:1278-84.

11 O'Brien PC, Fleming TR. A multiple testing procedure for clinical trials. Biometrics 1979;35:549-56.

12 Braun AB, Gibbons FK, Litonjua AA, et al. Low serum 25 -hydroxyvitamin $D$ at critical care initiation is associated with increased mortality. Crit Care Med 2012;40:63-72.

13 Moraes RB, Friedman G, Wawrzeniak IC, et al. Vitamin D deficiency is independently associated with mortality among critically ill patients. Clinics (Sao Paulo) 2015;70:326-32.

14 Amrein K, Zajic P, Schnedl C, et al. Vitamin D status and its association with season, hospital and sepsis mortality in critical illness. Crit Care 2014;18:R47.

15 Lee P, Eisman JA, Center JR. Vitamin D deficiency in critically ill patients. N Engl J Med 2009;360:1912-4.

16 Venkatram S, Chilimuri S, Adrish M, et al. Vitamin D deficiency is associated with mortality in the medical intensive care unit. Crit Care $2011 ; 15$.

17 Arnson Y, Gringauz I, Itzhaky D, et al. Vitamin D deficiency is associated with poor outcomes and increased mortality in severely ill patients. QJM 2012;105:633-9.

18 McNally JD, Menon K. Vitamin D deficiency in surgical congenital heart disease: prevalence and relevance. Trans/ Pediatr 2013;2:99-111.

19 Bjelakovic G, Gluud LL, Nikolova D, et al. Vitamin D supplementation for prevention of mortality in adults. Cochrane Database Syst Rev 2011:CD007470.

20 Rejnmark L, Avenell A, Masud T, et al. Vitamin D with calcium reduces mortality: patient level pooled analysis of 70,528 patients from eight major vitamin D trials. J Clin Endocrinol Metab 2012;97:2670-81.

21 Aspelund T, Grübler MR, Smith AV, et al. Effect of genetically low 25-hydroxyvitamin D on mortality risk: Mendelian randomization analysis in 3 large European cohorts. Nutrients 2019;11:E74.

22 Amrein K, Litonjua AA, Moromizato T, et al. Increases in prehospitalization serum $25(\mathrm{OH}) \mathrm{D}$ concentrations are associated with improved 30-day mortality after hospital admission: A cohort study. Clin Nutr 2016;35:514-21.

23 Han JE, Alvarez JA, Jones JL, et al. Impact of high-dose vitamin $\mathrm{D}_{3}$ on plasma free 25-hydroxyvitamin $\mathrm{D}$ concentrations and antimicrobial peptides in critically ill mechanically ventilated adults. Nutrition 2017;38:102-8.

24 European Medicines Agency. Clinical investigation of medicinal products for the treatment of sepsis. Reference: CPMP/ EWP/4713/2003.

25 European Medicines Agency.. Clinical investigation of medicinal products in the treatment of patients with acute respiratory distress syndrome. Reference: CPMP/EWP/504/97 Rev.1. 